Additional services for Journal of Fluid Mechanics:

Email alerts: $\underline{\text { Click here }}$

Subscriptions: $\underline{\text { Click here }}$

Commercial reprints: $\underline{\text { Click here }}$

Terms of use : $\underline{\text { Click here }}$

\title{
Trapped modes in a waveguide with a long obstacle
}

N. S. A. KHALLAF, L. PARNOVSKI and D. VASSILIEV

Journal of Fluid Mechanics / Volume 403 / January 2000, pp 251 - 261

DOI: 10.1017/S0022112099007028, Published online: 08 September 2000

Link to this article: http://journals.cambridge.org/abstract_S0022112099007028

How to cite this article:

N. S. A. KHALLAF, L. PARNOVSKI and D. VASSILIEV (2000). Trapped modes in a waveguide with a long obstacle. Journal of Fluid Mechanics, 403, pp 251-261 doi:10.1017/S0022112099007028

Request Permissions : $\underline{\text { Click here }}$ 


\title{
Trapped modes in a waveguide with a long obstacle
}

\author{
By N. S. A. KHALLAF ${ }^{1} \dagger$, L. PARNOVSKI \\ AND D. VASSILIEV \\ ${ }^{1}$ University of King Abdulaziz, College of Education, Department of Mathematics, Al-Madinah \\ Al-Munawwarah, PO Box 344, Kingdom of Saudi Arabia \\ ${ }^{2}$ School of Mathematical Sciences, University of Sussex, Brighton BN1 9QH, UK \\ ${ }^{3}$ Department of Mathematical Sciences, University of Bath, Claverton Down, \\ Bath BA2 7AY, UK
}

(Received 7 October 1998 and in revised form 13 September 1999)

Consider an infinite two-dimensional acoustic waveguide containing a long rectangular obstacle placed symmetrically with respect to the centreline. We search for trapped modes, i.e. modes of oscillation at particular frequencies which decay down the waveguide. We provide analytic estimates for trapped mode frequencies and prove that the number of trapped modes is asymptotically proportional to the length of the obstacle.

\section{Introduction}

In this paper we consider an infinite planar waveguide containing an obstacle. We assume that the waveguide is occupied by an acoustic medium and study the free vibrations of this medium. Mathematically the problem is described by the Helmholtz equation for the potential of displacements $\phi(x, y)$, subject to Neumann boundary conditions on the sides of the waveguide and on the boundary of the obstacle (hard walls). The same mathematical problem appears in the study of water waves in channels; see, for example, Evans \& Linton (1991) and Callan, Linton \& Evans (1991).

If for a particular vibration frequency the problem has a non-trivial solution decaying at infinity, we shall say that we have a trapped mode. As pointed out in Evans, Levitin \& Vassiliev (1994), Roitberg, Vassiliev \& Weidl (1998) and Davies \& Parnovski (1998) the existence of trapped modes is usually related to certain symmetries in the problem. In this paper we deal with the most basic type of symmetry, namely when the obstacle is symmetric about the centreline. We restrict ourselves to the study of antisymmetric modes, and search for trapped mode eigenvalues below the first antisymmetric cutoff.

It was proved in Evans et al. (1994) that for a fairly general symmetric obstacle there is at least one trapped mode. The purpose of this paper is to study what happens when the obstacle is long. For definiteness we assume throughout the paper (apart from the last section) that the obstacle is a rectangle of length $2 a>0$ and width $0 \leqslant 2 b<2$ ( $b=0$ corresponds to an infinitely thin rigid plate). Obstacles in the shape of rectangular blocks or plates are standard test cases studied previously

$\dagger$ Present address: School of Mathematical Sciences, University of Sussex, Brighton BN1 9QH, UK. 


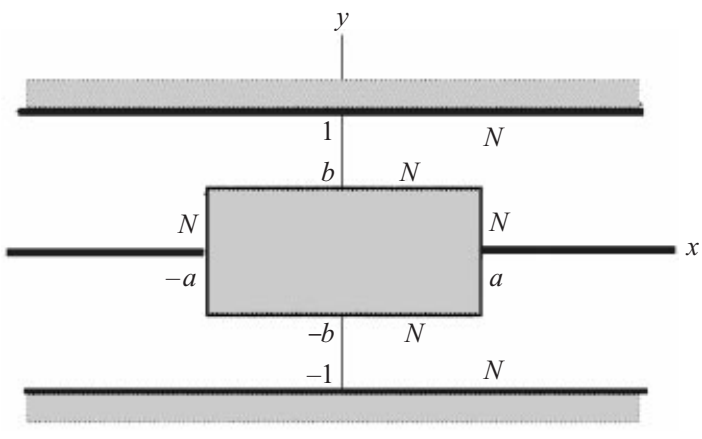

Figure 1. Domain $\tilde{\Omega}$.

by other authors, see, for example, Evans \& Linton (1991), Evans (1992) and Evans \& Linton (1992).

The main result of our paper is that as $a \rightarrow+\infty$ the total number of trapped modes is asymptotically equal to $a$ (see Corollary 3 in $\S 3$ ). Thus, for a long rectangular obstacle the total number of trapped modes is asymptotically determined only by the length of the obstacle. Moreover, we prove (Theorem 5.1) that the same is true for long obstacles of fairly general shape. Numerical results are given in $\S 4$.

We were to a large extent motivated by Evans \& Linton (1991), Evans (1992) and Evans \& Linton (1992). Though the numerical results of these authors do not extend to very long obstacles, they helped us predict the asymptotics. Moreover, for $b=0$ our asymptotics can be obtained directly from Evans (1992).

\section{Statement of the problem}

Let $\tilde{\Omega}$ be the unbounded planar domain defined as

$$
\tilde{\Omega}=\{(-\infty, \infty) \times(-1,1)\} \backslash\{[-a, a] \times[-b, b]\}
$$

(see figure 1), where $a>0$ and $0 \leqslant b<1$. We are interested in finding trapped modes, i.e. eigenvalues of the Neumann Laplacian in $\tilde{\Omega}$. In other words, we are looking for the values of $\lambda$ for which there exists a non-trivial square-integrable solution $\phi(x, y)$ of the Helmholtz equation

$$
-\Delta \phi=\lambda \phi \quad \text { in } \tilde{\Omega}
$$

subject to the boundary conditions

$$
\left.\frac{\partial \phi}{\partial n}\right|_{\partial \tilde{\Omega}}=0
$$

(note that the boundary $\partial \tilde{\Omega}$ is the union of the boundary of the strip and the boundary of the obstacle). The spectral parameter $\lambda$ is related to the vibration frequency $\omega$ as $\lambda=\omega^{2} / c^{2}$, where $c$ is the speed of sound in the medium.

It is a standard fact (see, e.g., Evans et al. 1994) that the spectrum of the problem $(2.1)-(2.2)$ is the non-negative semi-axis $[0,+\infty)$. Thus, the whole spectrum is essential. Moreover, it is known that the spectrum consists of the absolutely continuous part and, possibly, of eigenvalues of finite multiplicity which can accumulate only at infinity. Therefore, eigenvalues (if any) are embedded in the continuous spectrum. In general, finding such eigenvalues (both numerically and theoretically) is not an easy 


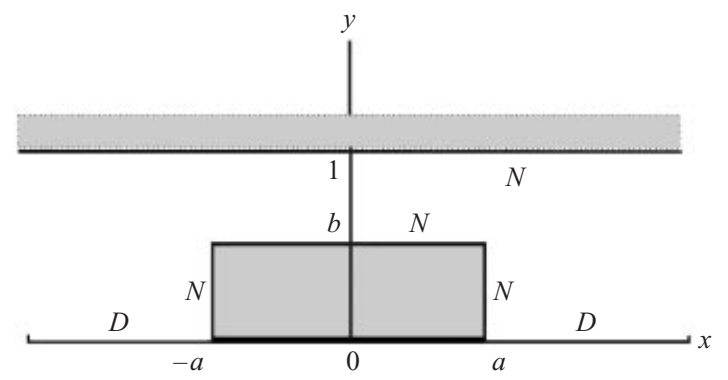

FIGURE 2. Domain $\Omega^{+}$.

problem because they are extremely unstable and can be destroyed by an arbitrarily small perturbation.

However, in our special case the situation is simplified because of the symmetry; see Evans et al. (1994), Roitberg et al. (1998) and Davies \& Parnovski (1998) for a discussion of the notion of symmetry in this setting. Namely, let us consider functions which are odd in $y$ :

$$
\mathscr{H}_{1}:=\left\{\phi \in L^{2}(\tilde{\Omega}) \mid \phi(x,-y)=-\phi(x, y)\right\} .
$$

One can easily check that $\mathscr{H}_{1}$ is an invariant subspace of $L^{2}(\tilde{\Omega})$ with respect to the action of $-\Delta$. Moreover, the essential spectrum of $-\left.\Delta\right|_{\mathscr{H}_{1}}$ is the interval $\left[\pi^{2} / 4, \infty\right)$. Therefore, the eigenvalues of $-\left.\Delta\right|_{\mathscr{H}_{1}}$ lying in $\left[0, \pi^{2} / 4\right)$ are stable under small perturbations, and one can find such eigenvalues using standard variational methods. The spectral problem for $-\left.\Delta\right|_{\mathscr{H}_{1}}$ is equivalent to the spectral problem for the Laplace operator in

$$
\Omega^{+}:=\{(x, y) \in \tilde{\Omega} \mid y>0\}
$$

with Neumann boundary conditions on the 'old' boundary and Dirichlet boundary conditions on the 'new' boundary $\{(x, y)|y=0| x \mid,>a\}$, see figure 2. Hence, the problem is reduced to finding values of $\lambda$ for which there is a non-trivial square integrable $\phi(x, y)$ satisfying the Helmholtz equation

$$
-\Delta \phi=\lambda \phi \quad \text { in } \Omega^{+}
$$

subject to the boundary conditions

$$
\begin{gathered}
\left.\frac{\partial \phi}{\partial y}\right|_{y=1}=0, \\
\left.\frac{\partial \phi}{\partial y}\right|_{|x|<a, y=b}=0, \\
\left.\frac{\partial \phi}{\partial x}\right|_{|x|=a, 0<y<b}=0, \\
\left.\phi\right|_{|x|>a, y=0}=0 .
\end{gathered}
$$

All the eigenvalues of (2.3)-(2.7) lying in $\left[0, \pi^{2} / 4\right)$ can be found using the variational approach. Put

$$
Q(\phi):=\frac{\iint_{\Omega^{+}}|\nabla \phi|^{2} \mathrm{~d} x \mathrm{~d} y}{\iint_{\Omega^{+}}|\phi|^{2} \mathrm{~d} x \mathrm{~d} y},
$$




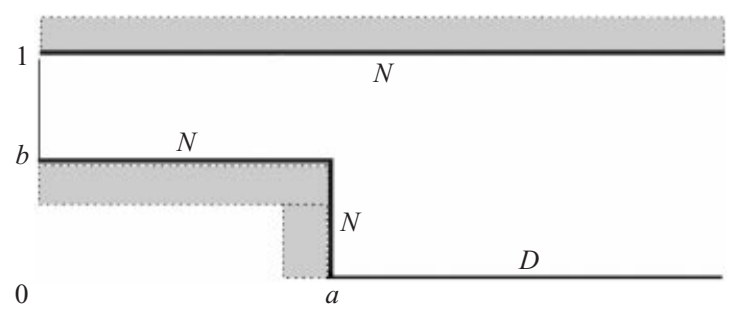

Figure 3. Domain $\Omega$.

$$
\lambda_{1}:=\inf Q(\phi),
$$

where the infimum is taken over functions $\phi \not \equiv 0$ satisfying the boundary condition (2.7) (here we do not have to impose the Neumann conditions (2.4)-(2.6) because they are automatically satisfied at the extrema of $Q(\phi))$. Now, if $\lambda_{1}<\pi^{2} / 4$, then $\lambda_{1}$ is the first eigenvalue of (2.3)-(2.7) and the function $\phi_{1}$ on which the infimum is attained is the eigenfunction corresponding to $\lambda_{1}$. (It is easy to see that $\lambda_{1}>0$.) Analogously, if $\lambda_{2}:=\inf _{\phi \perp \phi_{1}} Q(\phi)<\pi^{2} / 4$, then $\lambda_{2}$ is the second eigenvalue of (2.3)-(2.7) and the function $\phi_{2}$ on which the infimum is attained is the eigenfunction corresponding to $\lambda_{2}$. This procedure can be repeated until we get $\lambda_{k+1}=\pi^{2} / 4$. Then we will know that in the interval $\left(0, \pi^{2} / 4\right)$ there are exactly $k$ eigenvalues of the problem (2.3)-(2.7) and these eigenvalues are $\lambda_{1}, \ldots, \lambda_{k}$. However, we still will not be able to say anything about eigenvalues lying in $\left[\pi^{2} / 4, \infty\right)$. To find them one should either use a different approach, or find a new symmetry which will move the continuous spectrum further up.

In our case there is another obvious symmetry-reflection with respect to the $y$ axis. This symmetry does not change the continuous spectrum, but nevertheless makes computations easier. So let us consider the two subspaces

$$
\mathscr{H}^{ \pm}:=\left\{\phi \in L^{2}\left(\Omega^{+}\right) \mid \phi(-x, y)= \pm \phi(x, y)\right\} .
$$

These subspaces are invariant under the action of $-\Delta$. Therefore, the spectrum of the problem (2.3)-(2.7) is the union of the spectra of the restrictions of (2.3)-(2.7) to $\mathscr{H}^{+}$ and $\mathscr{H}^{-}$. Moreover, the spectrum of (2.3)-(2.7) restricted to $\mathscr{H}^{+}\left(\mathscr{H}^{-}\right)$coincides with the spectrum of $-\Delta$ considered in

$$
\Omega:=\left\{(x, y) \in \Omega^{+} \mid x>0\right\}
$$

with unchanged boundary conditions on the 'old' boundary and Neumann (Dirichlet) boundary conditions on the 'new' boundary $\{(x, y) \mid x=0, b<y<1\}$, see figure 3 .

The continuous spectra of both these problems are still $\left[\pi^{2} / 4, \infty\right)$, but using the variational approach described above one can find the eigenvalues of these problems below $\pi^{2} / 4$. Let us call such eigenvalues $\lambda_{1}^{N}, \ldots, \lambda_{k_{N}}^{N}$ and $\lambda_{1}^{D}, \ldots, \lambda_{k_{D}}^{D}$ respectively, so that there are $k_{N}\left(k_{D}\right)$ eigenvalues of these problems below $\pi^{2} / 4$.

\section{Explicit estimates for eigenvalues}

The aim of this section is to prove the following.

Theorem 3.1. Let $\lambda_{j}^{N}, \lambda_{j}^{D} \in\left(0, \pi^{2} / 4\right)$ be the eigenvalues defined above. Then the following inequalities hold for all $j$ :

$$
\frac{\pi^{2}}{a^{2}}(j-1)^{2} \leqslant \lambda_{j}^{N} \leqslant \frac{\pi^{2}}{a^{2}}\left(j-\frac{1}{2}\right)^{2}
$$


and

$$
\frac{\pi^{2}}{a^{2}}\left(j-\frac{1}{2}\right)^{2} \leqslant \lambda_{j}^{D} \leqslant \frac{\pi^{2}}{a^{2}} j^{2} .
$$

Moreover, each interval (3.1), (3.2) with right endpoint $<\pi^{2} / 4$ contains one and only one eigenvalue.

Before giving the proof of this theorem let us state its immediate implications.

We define the counting function $N^{N}(\lambda)\left(N^{D}(\lambda)\right)$ to be the number of eigenvalues $\lambda_{j}^{N}$ $\left(\lambda_{j}^{D}\right)$ which are less than $\lambda$. We also define $N^{\text {total }}:=N^{N}\left(\pi^{2} / 4\right)+N^{D}\left(\pi^{2} / 4\right)=k_{N}+k_{D}$. In other words, $N^{\text {total }}$ is the total number of trapped modes antisymmetric with respect to the centreline with frequencies below the cutoff frequency (the frequency above which one gets propagating antisymmetric modes).

In what follows $[z]$ stands for the greatest integer strictly less than $z$; that is, $[z]$ is the integer part of $z$ if $z$ is non-integer, and $[z]=z-1$ if $z$ is integer. Theorem 3.1 implies

Corollary 1. Let $\lambda \in\left(0, \pi^{2} / 4\right]$. Then

$$
\left[\frac{a}{\pi} \lambda^{1 / 2}\right] \leqslant N^{D}(\lambda) \leqslant N^{N}(\lambda) \leqslant\left[\frac{a}{\pi} \lambda^{1 / 2}\right]+1 .
$$

COROllary 2. Let $\lambda \in\left(0, \pi^{2} / 4\right]$ be fixed. Then the following asymptotic formulae hold as $a \rightarrow+\infty$ :

$$
N^{N}(\lambda) \sim \frac{a}{\pi} \lambda^{1 / 2}, \quad N^{D}(\lambda) \sim \frac{a}{\pi} \lambda^{1 / 2}
$$

COROLlary 3. The following asymptotic formula for the total number of trapped modes holds as $a \rightarrow+\infty$ :

$$
N^{\text {total }} \sim a
$$

Proof of Theorem 3.1. Let us prove formula (3.1) (formula (3.2) is proved similarly). Throughout the proof we will be dropping the superscript $N$ referring to the type of boundary condition on the $y$-axis. In particular, we shall write $N(\lambda)=N^{N}(\lambda)$. The number $\lambda$ will be assumed to satisfy $0<\lambda<\pi^{2} / 4$.

The main idea is to use Dirichlet-Neumann bracketing along the line $x=a$. Let us cut $\Omega$ into two domains: $A:=\{(x, y) \in \Omega \mid x<a\}$ and $B:=\{(x, y) \in \Omega \mid x>a\}$. Denote by $\lambda_{j}^{A, N}\left(\lambda_{j}^{A, D}\right)$ the eigenvalues of $-\Delta$ acting in the domain $A$ with Neumann (Dirichlet) boundary conditions on the 'new' boundary $\{(x, y) \mid x=a, b<y<1\}$ and the same boundary conditions on the 'old' boundary as before; of course, the latter means having Neumann boundary conditions on the top, bottom, and left-hand sides of $A$. In the same way we define $\lambda_{j}^{B, N}$ and $\lambda_{j}^{B, D}$. Let $N_{A}^{N}(\lambda)$ be the number of eigenvalues $\lambda_{j}^{A, N}$ which are less than $\lambda$; similarly we define the three other counting functions. Let us emphasize that now the letters $N$ and $D$ stand for boundary conditions on the interval $x=a, b<y<1$, and not on the $y$-axis!

The essential spectrum of all the operators involved is either empty $(-\Delta$ acting on $A)$, or $\left[\pi^{2} / 4, \infty\right)(-\Delta$ acting on $B$ or $\Omega)$. We have assumed that $\lambda<\pi^{2} / 4$, therefore the variational principle implies the following formula, known as the Dirichlet-Neumann bracketing (see e.g. Courant \& Hilbert, Theorem VI.2.5):

$$
N_{A}^{D}(\lambda)+N_{B}^{D}(\lambda) \leqslant N(\lambda) \leqslant N_{A}^{N}(\lambda)+N_{B}^{N}(\lambda) .
$$




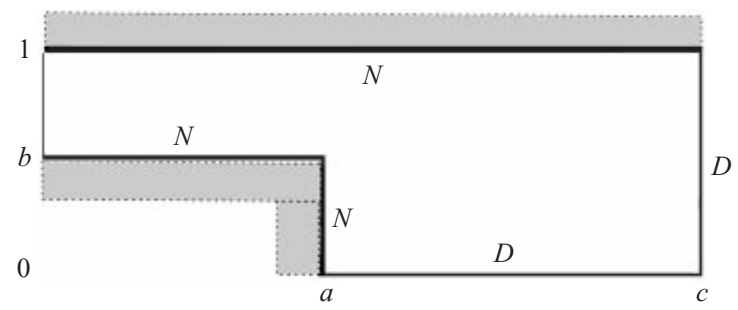

FIGURE 4. Truncated domain $\Omega$.

Using separation of variables one can easily compute the eigenvalues on $A$ : say, if we have Dirichlet conditions on the right-hand side, then

$$
\left\{\lambda_{j}^{A, D}\right\}_{j=1}^{\infty}=\left\{\frac{\pi^{2}}{a^{2}}\left(n-\frac{1}{2}\right)^{2}+\frac{\pi^{2}}{(1-b)^{2}}(m-1)^{2}\right\}_{n, m=1}^{\infty} .
$$

In a similar way

$$
\left\{\lambda_{j}^{A, N}\right\}_{j=1}^{\infty}=\left\{\frac{\pi^{2}}{a^{2}}(n-1)^{2}+\frac{\pi^{2}}{(1-b)^{2}}(m-1)^{2}\right\}_{n, m=1}^{\infty} .
$$

Note that the values of the expressions in the right-hand sides of (3.7)-(3.8) corresponding to $m>1$ are greater than $\pi^{2} / 4$, therefore, when dealing with eigenvalues below $\pi^{2} / 4$, we may assume that $m=1$. Let us now look at the sets $\left\{\lambda_{j}^{B, N}\right\} \cap\left[0, \pi^{2} / 4\right)$ and $\left\{\lambda_{j}^{B, D}\right\} \cap\left[0, \pi^{2} / 4\right)$. Using separation of variables one can easily show that the first of these two sets is empty. Indeed, the spectrum of $-\Delta$ on $B$ with Neumann boundary conditions on the top and left-hand sides and Dirichlet boundary condition on the bottom side is the interval $\left[\pi^{2} / 4, \infty\right)$. Let us now prove that $\left\{\lambda_{j}^{B, D}\right\} \cap\left[0, \pi^{2} / 4\right)=\emptyset$. Suppose that $\lambda_{1}^{B, D}<\pi^{2} / 4$. Then the variational principle implies $\lambda_{1}^{B, N}<\pi^{2} / 4$, and we have just seen that this is not the case. Therefore, for $\lambda<\pi^{2} / 4$ we have

$$
N_{A}^{D}(\lambda) \leqslant N(\lambda) \leqslant N_{A}^{N}(\lambda) \text {. }
$$

Formulae (3.7)-(3.9) imply (3.1).

\section{Numerical results}

In this section we provide some numerical results for the eigenvalues $\lambda_{j}^{N}$ and $\lambda_{j}^{D}$ introduced at the end of $\S 2$.

Computations were done using the variational method. Namely, we considered the Rayleigh quotient $Q(\phi)$ on $\Omega$ (see (2.8)) and, minimizing it, successively determined the eigenvalues and eigenfunctions. Applying this procedure we imposed Dirichlet boundary conditions on the appropriate parts of $\partial \Omega$, but did not impose Neumann ones because they are automatically satisfied at the extrema of $Q(\phi)$. The discretization of $Q(\phi)$ was carried out over a square mesh with mesh size $h=1 / 20$. We truncated our domain $\Omega$ to a finite one by imposing a Dirichlet boundary condition at $x=c=40$, see figure 4. (Our numerical calculations showed that the dependence of the eigenvalues on the choice of $c$ is hardly noticeable for $c \geqslant 40$.) The actual minimization of the (discretised) $Q(\phi)$ was performed by the gradient method. As the first approximation for the $n$th eigenfunction we took

$$
\phi(x, y)= \begin{cases}\sin (n / a) \pi x & \text { for } x \leqslant a \\ 0 & \text { for } x \geqslant a\end{cases}
$$




\begin{tabular}{|c|c|c|c|c|c|c|c|}
\hline$a$ & 1 & 2 & 3 & 4 & 5 & 10 & 20 \\
\hline$\lambda_{1}^{N}$ & 1.102 & 0.410 & 0.209 & 0.126 & 0.085 & 0.023 & 0.006 \\
\hline$\lambda_{2}^{N}$ & & & 1.780 & 1.108 & 0.748 & 0.206 & 0.053 \\
\hline$\lambda_{3}^{N}$ & & & & & 2.016 & 0.568 & 0.149 \\
\hline$\lambda_{4}^{N}$ & & & & & & 1.108 & 0.289 \\
\hline$\lambda_{5}^{N}$ & & & & & & 1.818 & 0.477 \\
\hline$\lambda_{6}^{N}$ & & & & & & & 0.712 \\
\hline$\lambda_{7}^{N}$ & & & & & & & 0.994 \\
\hline$\lambda_{8}^{N}$ & & & & & & & 1.320 \\
\hline$\lambda_{9}^{N}$ & & & & & & & 1.690 \\
\hline$\lambda_{10}^{N}$ & & & & & & & 2.100 \\
\hline$\lambda_{1}^{D}$ & & 1.554 & 0.821 & 0.500 & 0.335 & 0.092 & 0.024 \\
\hline$\lambda_{2}^{D}$ & & & & 1.920 & 1.313 & 0.365 & 0.094 \\
\hline$\lambda_{3}^{D}$ & & & & & & 0.816 & 0.218 \\
\hline$\lambda_{4}^{D}$ & & & & & & 1.442 & 0.373 \\
\hline$\lambda_{5}^{D}$ & & & & & & 2.234 & 0.589 \\
\hline$\lambda_{6}^{D}$ & & & & & & & 0.847 \\
\hline$\lambda_{7}^{D}$ & & & & & & & 1.151 \\
\hline$\lambda_{8}^{D}$ & & & & & & & 1.500 \\
\hline$\lambda_{9}^{D}$ & & & & & & & 1.891 \\
\hline$\lambda_{10}^{D}$ & & & & & & & 2.315 \\
\hline
\end{tabular}

in the case of the Dirichlet boundary condition on the $y$-axis, and

$$
\phi(x, y)= \begin{cases}\cos \left(\left(n-\frac{1}{2}\right) / a\right) \pi x & \text { for } x \leqslant a \\ 0 & \text { for } x \geqslant a\end{cases}
$$

in the case of the Neumann one. It turns out that the graphs of the actual eigenfunctions (which we omit for the sake of brevity) are remarkably close to (4.1) and (4.2).

Our main numerical results are presented in tables 1 and 2. Of course, for $a$ that are not too large these results agree with those of Evans \& Linton (1991). The accuracy of our numerical results is determined by two main factors: the choice of truncation distance $c$ and mesh size $h$. Elementary analysis involving separation of variables shows that imposing an artificial boundary condition at $x=c$ introduces an error of the order of $\mathrm{e}^{-\sqrt{\pi^{2} / 4-\lambda_{j}}(c-a)}$ which is negligible for our values of $a, c$ and $\lambda_{j}$. Predicting the error arising from the choice of mesh size is more complicated, but our numerical experiments with the refinement from $h=1 / 10$ to $h=1 / 20$ indicate that the relative error in the determination of the $\lambda_{j}$ is not greater than $1 \%$.

Our results seem to suggest that for large values of $a$ the dependence of eigenvalues on $b$ is insignificant. This agrees with the fact that the inequalities (3.1), (3.2) do not contain the parameter $b$. Note that a rigorous analysis of this $b$-dependence is a delicate mathematical problem because changing $b$ involves moving the part of 


$\begin{array}{cccccccc}a & 1 & 2 & 3 & 4 & 5 & 10 & 20 \\ \lambda_{1}^{N} & 1.494 & 0.484 & 0.234 & 0.137 & 0.089 & 0.024 & 0.006 \\ \lambda_{2}^{N} & & & 2.237 & 1.295 & 0.841 & 0.216 & 0.055 \\ \lambda_{3}^{N} & & & & & 2.330 & 0.601 & 0.152 \\ \lambda_{4}^{N} & & & & & & 1.178 & 0.297 \\ \lambda_{5}^{N} & & & & & & 1.946 & 0.493 \\ \lambda_{6}^{N} & & & & & & & 0.731 \\ \lambda_{7}^{N} & & & & & & & 1.023 \\ \lambda_{8}^{N} & & & & & & & 1.362 \\ \lambda_{9}^{N} & & & & & & & 1.746 \\ \lambda_{10}^{N} & & & & & & & 2.186 \\ \lambda_{1}^{D} & & 1.888 & 0.925 & 0.545 & 0.359 & 0.094 & 0.024 \\ \lambda_{2}^{D} & & & 2.299 & 1.496 & 0.385 & 0.097 \\ \lambda_{3}^{D} & & & & & & 0.865 & 0.219 \\ \lambda_{4}^{D} & & & & & & 1.538 & 0.388 \\ \lambda_{5}^{D} & & & & & & 2.404 & 0.606 \\ \lambda_{6}^{D} & & & & & & & 0.872 \\ \lambda_{7}^{D} & & & & & & & 1.186 \\ \lambda_{8}^{D} & & & & & & & 1.550 \\ \lambda_{9}^{D} & & & & & & & 1.962 \\ \lambda_{10}^{D} & & & & & & & 2.423\end{array}$

TABLE 2. Eigenvalues for $b=1 / 2$.

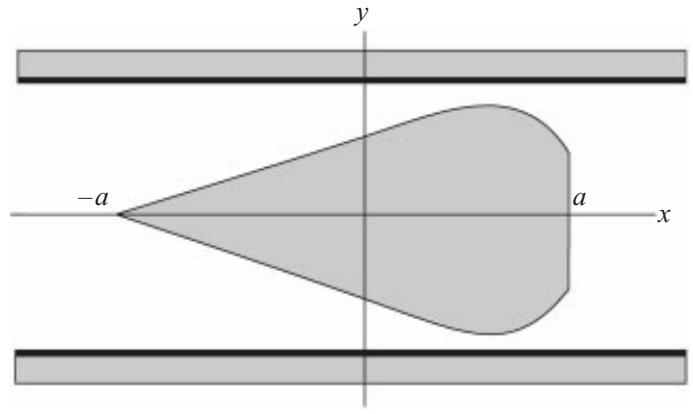

FiguRE 5. Obstacle of fairly general shape.

the boundary with the Neumann condition. It is known that in such a situation the eigenvalues are not, in general, domain monotone.

Table 3 provides a numerical illustration of Theorem 3.1. This table indicates that our upper estimate is in most cases closer to the actual eigenvalue than the lower one.

\section{Long obstacles of general shape}

Let $-1=X_{0}<X_{1}<\cdots<X_{m}=1$ be a partition of the interval $[-1,1]$ into $m$ subintervals $\left[X_{i-1}, X_{i}\right]$, and let $f_{i}:\left[X_{i-1}, X_{i}\right] \rightarrow[0,1)$ be a family of smooth functions. 


$\begin{array}{ccccc}\left(\pi^{2} / a^{2}\right)(j-1)^{2} & \lambda_{j}^{N} & \left(\pi^{2} / a^{2}\right)\left(j-\frac{1}{2}\right)^{2} & \lambda_{j}^{D} & \left(\pi^{2} / a^{2}\right) j^{2} \\ 0.000 & 0.005 & 0.006 & 0.024 & 0.025 \\ 0.025 & 0.053 & 0.056 & 0.094 & 0.099 \\ 0.099 & 0.149 & 0.154 & 0.218 & 0.222 \\ 0.222 & 0.289 & 0.302 & 0.373 & 0.395 \\ 0.394 & 0.477 & 0.499 & 0.589 & 0.617 \\ 0.617 & 0.712 & 0.746 & 0.847 & 0.888 \\ 0.888 & 0.994 & 1.042 & 1.151 & 1.209 \\ 1.209 & 1.320 & 1.388 & 1.500 & 1.579 \\ 1.579 & 1.690 & 1.783 & 1.891 & 1.999 \\ 1.998 & 2.099 & 2.227 & 2.315 & 2.467\end{array}$

TABLE 3. Numerical illustration of Theorem $3.1(a=20, b=0)$.

Let $a>0$ be a real parameter. Put

$$
\mathcal{O}_{a}:=\bigcup_{i=1}^{m}\left\{(x, y) \mid x \in\left[a X_{i-1}, a X_{i}\right], 0 \leqslant y \leqslant f_{i}(x / a)\right\}
$$

(so that $\mathcal{O}_{a}$ is an obstacle of length $2 a$ ). Let us consider the problem (2.1) in the domain $\Omega_{a}^{+}:=(-\infty, \infty) \times(0,1) \backslash \mathcal{O}_{a}$ with Neumann boundary conditions on $\{y=1\} \cup \partial \mathcal{O}_{a}$ and Dirichlet boundary conditions on $\{|x|>a, y=0\}$. Figure 5 shows an example of such an obstacle. We denote by $N(\lambda)$ the number of eigenvalues of this problem below $\lambda$, and put $N^{\text {total }}:=N\left(\pi^{2} / 4\right)$. The aim of this section is to prove the following theorem which generalizes Corollaries 2 and 3 from $\S 3$.

Theorem 5.1. Let $\lambda \in\left(0, \pi^{2} / 4\right]$ be fixed. Then

$$
N(\lambda) \sim \frac{2 a}{\pi} \lambda^{1 / 2}
$$

as $a \rightarrow+\infty$. In particular,

$$
N^{\text {total }} \sim a
$$

Proof. In order to prove (5.1) we have to show that

$$
N(\lambda)>\frac{2 a \sqrt{\lambda}}{\pi}-o(a)
$$

and

$$
N(\lambda)<\frac{2 a \sqrt{\lambda}}{\pi}+o(a)
$$

We shall, as before, prove these estimates using Dirichlet-Neumann bracketing.

We first prove (5.3). Let $\varepsilon>0$ be a small number. Put

$$
\begin{gathered}
n_{i}=n_{i}(\varepsilon, a):=\left[\frac{a\left(X_{i}-X_{i-1}\right) \sqrt{\lambda-\varepsilon}}{\pi}\right], \quad i=1, \ldots, m, \\
x_{i, j}=x_{i, j}(\varepsilon, a):=a\left(X_{i-1}+\frac{j\left(X_{i}-X_{i-1}\right)}{n_{i}}\right), \quad i=1, \ldots, m, j=0,1, \ldots, n_{i},
\end{gathered}
$$


and impose additional Dirichlet conditions on the vertical intervals

$$
\left\{(x, y) \mid x=x_{i, j}, f_{i}(x / a)<y<1\right\}, \quad i=1, \ldots, m, j=0,1, \ldots, n_{i} .
$$

The variational principle implies that the spectrum of this new problem (i.e. the problem (2.1) with Neumann boundary conditions on $\{y=1\} \cup \partial \mathcal{O}$ and Dirichlet boundary conditions on $\{|x|>a, y=0\}$ and on (5.6)) is higher than that of the initial problem. In other words, $N(\lambda)$ can be estimated from below by the corresponding counting function of the new problem. The latter is, in turn, not less than

$$
\sum_{i=1}^{m} \sum_{j=1}^{n_{i}} N_{i, j}(\lambda)
$$

Here $N_{i, j}(\lambda)$ is the number of eigenvalues of the Laplacian on the 'almost rectangle'

$$
\Omega_{i, j}:=\left\{(x, y) \mid x_{i, j-1}<x<x_{i, j}, f_{i}(x / a)<y<1\right\}
$$

with Neumann boundary conditions on the horizontal sides $y=1$ and $y=f_{i}(x / a)$ and Dirichlet boundary conditions on the vertical sides $x=x_{i, j-1}$ and $x=x_{i, j}$. When $a \rightarrow \infty$ all 'almost rectangles' $\Omega_{i j}$ converge to proper rectangles of width $\pi / \sqrt{\lambda-\varepsilon}$, and the convergence is uniform in $i, j$. We now want to show that

$$
N_{i, j}(\lambda) \geqslant 1
$$

for large enough $a$ uniformly over $i, j$. In fact, it is easy to show (see, for example, Stollmann 1995) that the spectra of the $\Omega_{i j}$ converge to the spectra of proper rectangles (with corresponding boundary conditions) uniformly in $i, j$. Since the bottom eigenvalue of the proper rectangles is $\lambda-\varepsilon$, this would imply (5.7). To make the proof self-contained, we will also prove (5.7) directly. Indeed, consider the following test function $\phi_{i j}: \Omega_{i j} \rightarrow R$ :

$$
\phi_{i j}(x, y)=\sin \left(\frac{\pi\left(x-x_{i, j-1}\right)}{x_{i, j}-x_{i, j-1}}\right) .
$$

When $a \rightarrow \infty$, both expressions

$$
\iint_{\Omega_{i j}}\left|\nabla \phi_{i j}\right|^{2} \mathrm{~d} x \mathrm{~d} y
$$

and

$$
\iint_{\Omega_{i j}}\left|\phi_{i j}\right|^{2} \mathrm{~d} x \mathrm{~d} y
$$

converge to the corresponding integrals over the limiting rectangles uniformly over $i, j$. This means that their ratio

$$
Q\left(\phi_{i j}\right)=\frac{\iint_{\Omega_{i j}}\left|\nabla \phi_{i j}\right|^{2} \mathrm{~d} x \mathrm{~d} y}{\iint_{\Omega_{i j}}\left|\phi_{i j}\right|^{2} \mathrm{~d} x \mathrm{~d} y}
$$

uniformly converges to the corresponding expression with integrals taken over rectangles. Since the latter expression equals $\lambda-\varepsilon$, this argument shows that for large $a$ 
the inequality (5.7) is indeed fulfilled. Therefore,

$$
N(\lambda) \geqslant \sum_{i=1}^{m} \sum_{j=1}^{n_{i}} N_{i, j}(\lambda) \geqslant \sum_{i=1}^{m} \sum_{j=1}^{n_{i}} 1=\sum_{i=1}^{m} n_{i}>\frac{2 a \sqrt{\lambda-\varepsilon}}{\pi}-m=\frac{2 a \sqrt{\lambda}}{\pi}-m+O(\varepsilon a) .
$$

Since $\varepsilon>0$ is arbitrary, this implies (5.3).

The proof of (5.4) is analogous, the only difference being that we replace (5.5) by

$$
n_{i}=n_{i}(\varepsilon, a):=\left[\frac{a\left(X_{i}-X_{i-1}\right) \sqrt{\lambda+\varepsilon}}{\pi}\right]
$$

and impose Neumann conditions on the vertical intervals (5.6).

\section{REFERENCES}

Callan, M., Linton, C. M. \& Evans, D. V. 1991 Trapped modes in two-dimensional waveguides. J. Fluid Mech. 229, 51-64.

Courant, R. \& Hilbert, D. 1989 Methods of Mathematical Physics I. Wiley.

Davies, E. B. \& Parnovski, L. 1998 Trapped modes in acoustic waveguides. Q. J. Mech. Appl. Maths 51, 477-492.

Evans, D. V. 1992 Trapped acoustic modes. IMA J. Appl. Maths 49, 45-60.

Evans, D. V., Levitin, M. \& Vassiliev, D. 1994 Existence theorems for trapped modes. J. Fluid. Mech. 261, 21-31.

Evans, D. V. \& Linton, C. M. 1991 Trapped modes in open channels. J. Fluid. Mech. 225, 153-175.

Evans, D. V. \& Linton, C. M. 1992 Acoustic resonance in ducts. J. Sound Vib. 173, 85-94.

Roitberg, I., Vassiliev, D. \& Weidl, T. 1998 Edge resonance in an elastic semi-strip. Q. J. Mech. Appl. Maths 51, 1-13.

Stollmann, P. 1995 A convergence theorem for Dirichlet forms with applications to boundary value problems with varying domains. Math. Z. 219, 275-287. 